\section{|W!}

CSB

\title{
Developing a multidisciplinary strategy to interpret the impact of missense mutations in XPA on NER activity and cisplatin sensitivity
}

Alexandra M. Blee ${ }^{1,2}$, Bian Li ${ }^{3}$, Jens Meiler ${ }^{2}$, John A. Capra ${ }^{4}$, and Walter J. Chazin $1,2^{\star}$

1 Departments of Biochemistry and 2 Chemistry, Center for Structural Biology, Vanderbilt University, Nashville, TN 37235 4 Bakar Computational Health Sciences Institute and Department of Epidemiology and Biostatistics, University of California, San Francisco, CA 94107

1. Current efforts to predict the impact of VUS can be improved.

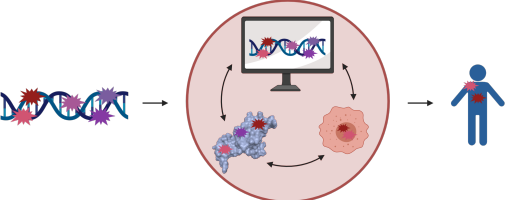

Predictive, correlative data is insufficient for robust evaluation of VUS.

Evidence-based validation is required to identify variants with clinical utility.

2. Nucleotide Excision Repair (NER) genes are mutated in cancers.

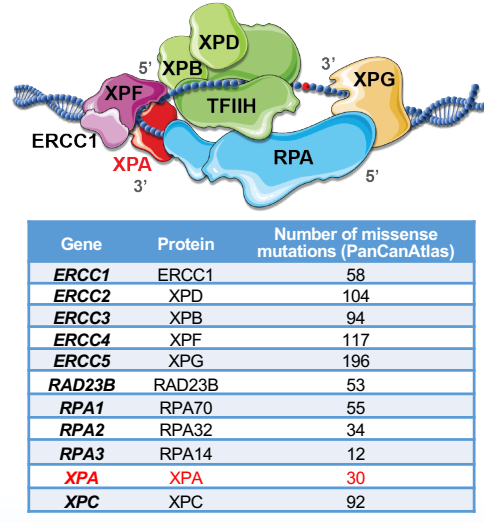

NER removes bulky DNA lesions formed by $\mathrm{UV}$ and cisplatin.

utations in ERCC2 correlate with improved parter cisplatin treatment. unknown.

\section{Pipeline to identify deleterious XPA mutants.} Input: candidate XPA mutation
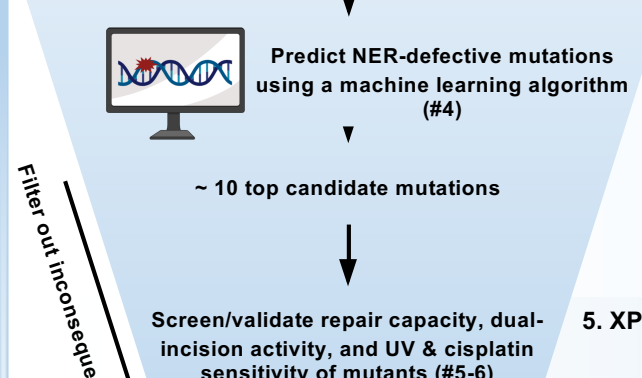

incision activity, and UV \& cisplati
sensitivity of mutants (\#5-6)

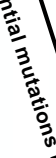

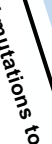

势

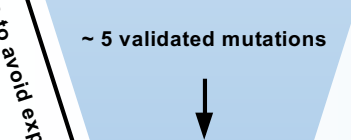

Characterize mutants

structurally (\#7-8)

苟

晋

竞
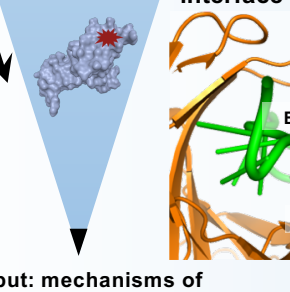

Output: mechanisms of NER dysfunction

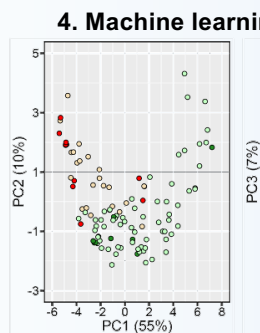

5. XPA mutations impact cell survival.

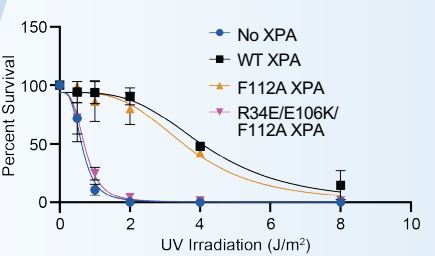

ells expressing NER-defective XPA mutants

have decreased survival after UV irradiation.

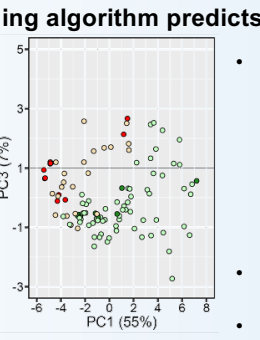

OKnown NER-proficient
O Predicted NER-proficient

6. XPA mutants impact localization to UV lesions.
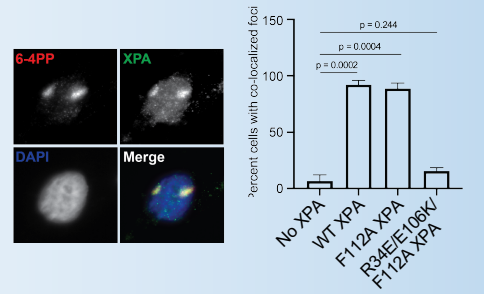

Cells expressing NER-defective XPA mutants have decreased co-localization with UV lesion

108 input mutants: 11 known NER-
defective

8 known NER-

competent literature and tumor genome databases 20 predictive each from web-based tools
Current strategies of VUS can be improved by inco

Future directions include:

Experimental validation of the machine learning algorithm

2) Cell-based, biophysical, and structura characterization of selected candidate mutations;

3) Expansion of the pipeline to other core NER proteins.

\section{Acknowledgements}

This research program is supported by $\mathrm{NIH}$ operating grants (R01 CA218315 and P01

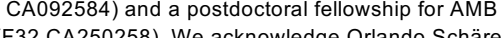
for sharing XP2OS cells and protocols and the for sharing XP2OS cells and protocols and the Biology for insights regarding variant effect

prediction.
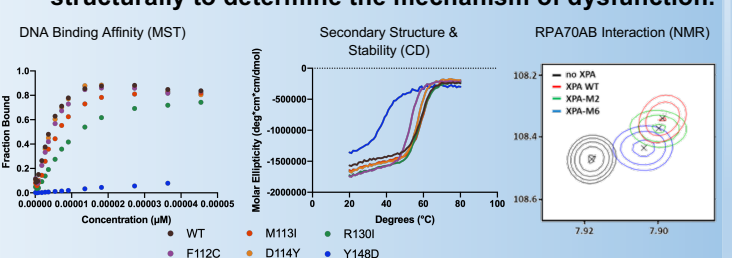

\section{References}

Knijnenburg, TA. et al. Cell Rep. 2018. Scharer, OD. Cold Spring Harb Perspect.

Sugitani, N. et al. DNA Repair. 2016. Van Allen, EM. et al. Cancer Discov. 2014 research. 2020. 